\title{
TEMPERED PROCESSES AND A RIESZ DECOMPOSITION FOR SOME MARTINGALES IN THE LIMIT
}

\author{
by LOUIS H. BLAKE
}

(Received 8 June, 1979)

\begin{abstract}
0. Introduction. Several papers have appeared in the past few years which have explored the topic of the Riesz decomposition for amarts. Such a decomposition for amarts enjoys several special properties. See [5, p. 208-209]. While it has been proved in [6] that not every martingale in the limit has a Riesz decomposition "in the weakest form assuring uniqueness" it is the major objective of this paper to characterize a class of martingales in the limit which is strictly larger than the class of amarts but enjoys all the properties of the decomposition for amarts.
\end{abstract}

In Section 1, the concept of tempered processes is introduced and explored. Tempered processes are at once a generalization of martingales and a major aspect of a Riesz decomposition for stochastic processes. A fundamental convergence theorem for tempered processes is presented.

In Section 2, a class of martingales in the limit is introduced which properly contains the class of amarts and also has a "complete" Riesz decomposition.

The following notation and definitions will be in use throughout the paper.

$(\Omega, \mathfrak{A}, P)$ is a probability space and $\left\{\mathfrak{I}_{n}\right\}_{n \geq 1}$ is an increasing sequence of sub sigma fields of $\mathfrak{U}$ to which (extended) real-valued processes will be adapted.

DefinItION. A processes $\left\{X_{n}\right\}_{n \geq 1}$ is

(i) martingale in the limit (MIL) if

$$
\lim _{m \rightarrow \infty} \sup _{n \geq m}\left|E\left(X_{n} \mid \mathfrak{A}_{m}\right)-X_{m}\right|=0
$$

(ii) amart if for each sequence of bounded increasing stopping times $\left\{\tau_{n}\right\}_{n \geq 1}$ with $\tau_{n} \uparrow \infty,\left\{E\left(X_{\tau_{n}}\right)\right\}_{n \geq 1}$ converges,

(iii) has a (weak) Riesz decomposition if it can be written as

$$
X_{n}=Y_{n}+Z_{n}
$$

where $\left\{Y_{n}\right\}_{n \geq 1}$ is martingale and for all $A \in \bigcup_{n=1}^{\infty} \mathfrak{U}_{n}$ we have $\int_{A} Z_{n} \rightarrow 0$.

NoTE. Weak Riesz decompositions are unique.

1. Tempered processes. Conditional expectation is often described as a smoothing operation. Once a given stochastic process is smoothed in some sense the resulting process might bear close or little resemblance to the original process. What will be done in this section is to introduce a smoothing procedure which is martingale-like in character and examine when the resulting process converges pointwise.

Glasgow Math. J. 22 (1981) 9-17. 
DEFINITION 1.1. Let $\left\{X_{n}\right\}_{n \geq 1}$ be an adapted process and suppose for each $m$

$$
\lim _{\substack{n \rightarrow \infty \\ n \geq m}} E\left(X_{n} \mid \mathfrak{A}_{m}\right)=Y_{m}, \quad \text { where } Y_{m} \text { is finite or infinite. }
$$

Then $\left\{X_{n}\right\}_{n \geq 1}$ will be called a temperable process.

DEFINITION 1.2. Let $\left\{Y_{m}\right\}_{m \geq 1}$ be a process for which there exists a process $\left\{X_{n}\right\}_{n \geq 1}$ where for each $m$

$$
\lim _{\substack{n \rightarrow \infty \\ n \geq m}} E\left(X_{n} \mid \mathfrak{A}_{m}\right)=Y_{m}
$$

Then $\left\{Y_{m}\right\}_{m \geq 1}$ will be called a tempered process.

It will often be convenient to say " $\left\{X_{n}\right\}_{n \geq 1}$ is tempered to $\left\{Y_{n}\right\}_{n \geq 1}$ " or " $\left\{Y_{n}\right\}_{n \geq 1}$ is tempered by $\left\{X_{n}\right\}_{n \geq 1}$."

Tempered processes abound with all sorts of convergence and non-convergence properties. The question arises: when do tempered processes converge pointwise?

Before an answer to this question is presented in the major theorem of this section, some examples and discussion of tempered processes is in order.

EXAMPLE 1.1. Every martingale is a tempered process. Inasmuch as not every martingale converges a.e. not every tempered process converges a.e. See, for example, Baez-Duarte [1].

EXAMPLE 1.2. Every amart is a temperable process which is tempered to a martingale. Here, too, the martingale to which the amart is tempered need not converge a.e. See ([5], p. 207 and p. 210).

EXAMPLE 1.3. Consider any sequence of independent random variables with same mean. Here is an example in which the tempered process could differ profoundly from the original; that is, the sequence of independent random variables is tempered to a constant. Convergence of the tempered process is assured!

EXAMPLE 1.4. Consider here:

$$
\begin{array}{ccc}
\Omega=[1, \infty), \quad A_{n} \equiv[n, n+1), & (n=1,2, \ldots) \\
\mathfrak{A}_{n} \equiv \sigma\left(A_{1}, A_{2}, \ldots, A_{n}\right) \text { and } & P\left(A_{n}\right) \equiv 1 / 2^{n} .
\end{array}
$$

Let $X_{n} \equiv I_{A_{1}}+I_{A_{n}}$. Then, for $n>m$,

$$
E\left(X_{n} \mid \mathfrak{A}_{m}\right)=\left\{\begin{array}{lll}
1 & \text { on } & A_{1} \\
0 & \text { on } & {[2, m+1)} \\
P\left(A_{n}\right) / P([m+1, \infty)) & \text { on } & {[m+1, \infty) .}
\end{array}\right.
$$

$Y_{m}=I_{A_{1}}$ for each $m$. Here the process $\left\{X_{n}\right\}_{n \geq 1}$ is tempered to a martingale while it is neither martingale nor is it sub or super martingale. The process $\left\{X_{n}\right\}_{n \geq 1}$ is martingale in 
the limit and amart inasmuch as $\sup _{n, n>m}\left|E\left(X_{n} \mid \mathfrak{A}_{m}\right)\right| \in L_{1}$ for each $m$. See [3] and [5], Proposition 2.2, respectively.

EXAMPLE 1.5. Consider the probability space of Example 1.4 and let

$$
\begin{gathered}
X_{n} \equiv I_{A_{1}}+2 I_{A_{2}}+\ldots+2^{n} I_{A_{n}} \\
E\left(X_{n+k} \mid \mathfrak{A}_{n}\right)=X_{n}+\frac{k}{P([n+1, \infty))} I_{[n+1, \infty)} .
\end{gathered}
$$

Here $Y_{n}=X_{n}$ on $[0, n+1)$ and $Y_{n}=\infty$ on $[n+1, \infty)$. It is clear that $\left\{X_{n}\right\}_{n \geq 1}$ is not amart but is MIL. Moreover, $\left\{Y_{n}-X_{n}\right\}_{n \geq 1}$ converges to zero a.e.; however, $\left\{Y_{n}\right\}_{n \geq 1}$ is not martingale.

It is natural to ask what relation temperable processes have to martingales.

Proposition 1.6. Let $\left\{X_{n}\right\}_{n \geq 1}$ be a temperable process tempered to $\left\{Y_{n}\right\}_{n \geq 1}$. Suppose $\sup \left|E\left(X_{n} \mid \mathfrak{U}_{m}\right)\right| \in L_{1}$ for each m. Then $\left\{Y_{n}\right\}_{n \geq 1}$ is a martingale.

Proof. Immediate.

The next theorem addresses a major question. If a process is tempered, when does it converge?

THEOREM 1.7. Suppose $\left\{X_{n}\right\}_{n \geq 1}$ is a process in $L_{1}$ tempered to $\left\{Y_{n}\right\}_{n \geq 1}$ and suppose $\left\{Y_{n}\right\}_{n \geq 1}$ is $L_{1}$ bounded. Then $\left\{Y_{n}\right\}_{n \geq 1}$ has a pointwise limit.

Proof. The proof will proceed by showing the number of downcrossings of an arbitrary interval must be finite and this will be sufficient in the context of the usual considerations in this type of argument. See [4].

Let $(a, b)$ be an arbitrary interval. Let $N$ be an arbitrary but fixed positive integer. Let $Y_{0} \equiv a$. Let $\left\{c_{i}\right\}_{i=1}^{\infty}$ be a sequence of positive reals with $\sum_{i=1}^{\infty} c_{i}<\infty$. Then define a finite sequence of stopping times relative to $(a, b)$ and $N$ as follows.

Let $T_{0} \equiv 0$ and let $T_{1}$ be the first integer $m$ with $N \geq m \geq T_{0}$ and

(i) $Y_{m} \geq b$,

(ii) $\left|E\left(X_{n} \mid \mathfrak{A}_{m}\right)-Y_{m}\right| \leq c_{1}$ for all $n \geq N$;

if none, let $T_{1}=N$, and $T_{2}$ be the first integer $m$ with $N \geq m>T_{1}$ and

(i) $Y_{m} \leq a$

(ii) $\left|E\left(X_{n} \mid \mathfrak{A}_{m}\right)-Y_{m}\right| \leq c_{2}$ for all $n \geq N$;

if none, let $T_{2}=N$, and so on up to $T_{N}=N$.

Consider

$$
Y_{N}-Y_{0}=Y_{T_{1}}-Y_{T_{0}}+Y_{T_{2}}-Y_{T_{1}}+\ldots+Y_{N}-Y_{T_{N-1}} .
$$

Let $\phi(N ; a, b)$ be the number of downcrossings relative to the above stopping times and observe that

$$
Y_{N}-Y_{0} \geq(b-a) \phi(N ; a, b)+\sum_{n=1} Y_{T_{2 n}}-Y_{T_{2 n-1}}, \quad \text { where } \quad 2 n \leq N
$$


Consider

$$
\begin{aligned}
\int\left(Y_{T_{2 n}}-Y_{T_{2 n-1}}\right) & =\sum_{k=1}^{N} \int_{\left\{T_{2 n}=k\right\}} Y_{k}-\sum_{k=1}^{N} \int_{\left\{T_{2 n-1}=k\right\}} Y_{k} \\
& =\sum_{k=1}^{N} \int_{\left\{T_{2 n}=k\right\}} Y_{k}-E\left(X_{N} \mid \mathfrak{A}_{k}\right)-\sum_{k=1}^{N} \int_{\left\{T_{2 n-1}=k\right\}} Y_{k}-E\left(X_{N} \mid \mathfrak{A}_{k}\right) \geq-c_{2 n}-c_{2 n-1} .
\end{aligned}
$$

So,

$$
\int Y_{N}-\int Y_{0} \geq(b-a) \int \phi(N ; a, b)-\sum_{k=1}^{N} c_{k} .
$$

Note that $\phi(N ; a, b)$ increases in $N$ and so, let $\phi(a, b) \equiv \lim _{N \rightarrow \infty} \phi(N ; a, b)$. It is clear that

$$
\sum_{k=1}^{\infty} c_{k}+\sup _{n=1}^{\infty} \int\left|Y_{n}\right|-a \geq(b-a) \int \phi(a, b) \geq 0 .
$$

Thus, $\phi(a, b)<\infty$ a.e. Let

$$
\Omega_{0}(a, b) \equiv\{\phi(a, b)<\infty) \cap \bigcap_{m=1}^{\infty}\left\{\lim _{\substack{n \rightarrow \infty \\ n \geq m}} E\left(X_{n} \mid \mathfrak{A}_{m}\right)=Y_{m}\right\}
$$

Let $D(a, b)$ be the number of downcrossings of the interval by the process $\left\{Y_{n}\right\}_{n \geq 1}$. Suppose for some $\omega \in \Omega_{0}(a, b)$ we have $D(a, b)(\omega)=\infty$. Then for a sufficiently large $N$ and a subsequence of these downcrossings it can be observed that $\phi(N: a, b)(\omega)>\phi(a, b)(\omega)$ and this is a contradiction. Thus $D(a, b)<\infty$ on the set $\Omega_{0}(a, b)$ and where $P\left(\Omega_{0}(a, b)\right)=1$. The proof is complete.

2. Riesz decomposition considerations for martingales in the limit. The topic of Riesz decomposition for amarts has been the subject of several articles. The most noteworthy of these for the purposes of this paper are [5], [6] and [10].

The major purpose of this section is to observe what properties of the Riesz decomposition for amarts are due to the fact that the process is amart and then characterize a strictly larger class of MIL which has all these important properties.

In [6, p. 317], a Riesz decomposition in "the weakest form assuring uniqueness" is a process $\left\{X_{n}\right\}_{n \geq 1}$ decomposed as $X_{n}=Y_{n}+Z_{n}$, where $\left\{Y_{n}\right\}_{n \geq 1}$ is a martingale and all that is asked of $\left\{Z_{n}\right\}_{n \geq 1}$ is that $\int_{A} Z_{n} \rightarrow 0$ as $n \rightarrow \infty$ for any $A \in \bigcup_{n=1}^{\infty} \mathfrak{A}_{n}$. (This is a natural extension of the decomposition of a supermartingale into a martingale and a Doob potential. See [7, p. 89].)

The first theorem states some sufficient conditions for an arbitrary process to have a Riesz decomposition in the weakest form assuring uniqueness.

THeOREM 2.1. Let $\left\{X_{n}\right\}_{x \geq 1}$ be a temperable process with $\sup \left|E\left(X_{n} \mid \mathfrak{U}_{m}\right)\right| \in L_{1}$ for each $m$. Then, $\left\{X_{n}\right\}_{n \geq 1}$ has a Riesz decomposition in the weakest form assuring uniqueness 
and further

(i) $\lim _{n \rightarrow \infty} E\left(Z_{n} \mid \mathfrak{A}_{m}\right)=0$ a.e. for each $m$,

(ii) $\lim _{n \rightarrow \infty} E\left(Z_{n} \mid \mathfrak{A}_{m}\right)=0$ in $L_{1}$ for each $m$ and

(iii) $\int \sup _{n \geq m}\left|E\left(Z_{n} \mid \mathfrak{U}_{m}\right)\right|<\infty$ for each $m$.

Proof. Immediate.

The following examples will illustrate among other things that there are readily available non-amarts which satisfy the hypothesis of Theorem 2.1 .

Example 2.1. A process which is not amart but is MIL is the following. Let $X_{n} \equiv n I_{A_{n}}$, where $\left\{A_{n}\right\}_{n \geq 1}$ is a sequence of pairwise independent sets and $\mathfrak{A}_{n} \equiv$ $\sigma\left(A_{1}, \ldots, A_{n}\right)$. Let $P\left(A_{n}\right)=1 / n^{2}$ for $n>1$ and $P\left(A_{1}\right)=0$. This example presented in [6, p. 318] is not amart (inasmuch as it does not satisfy the optional stopping theorem).

ExAmple 2.2. A process which is not MIL is the following. Let $\left\{X_{n}\right\}_{n \geq 1}$ be i.i.d. with mean zero and finite first moment. It is well-known that $P\left(X_{n}\right.$ converges $)=0$. If $\mathfrak{U}_{n}=$ $\sigma\left(X_{1}, \ldots, X_{n}\right)$, then the hypothesis of Theorem 2.1 . is easily satisfied. (Note that the process is not MIL in as much as an $L_{1}$ bounded MIL would have a pointwise limit. See [9].)

Hence, what aspects of the Riesz decomposition for amarts are due to the fact that the process is amart? In [5], Theorem 3.2 it follows that if the process is amart it satisfies the hypothesis of Theorem 2.1; but, moreover, the $\left\{Z_{n}\right\}_{n \geq 1}$ process is amart and has the additional convergence properties that $Z_{n} \rightarrow 0$ a.e. and in $L_{1}$ and $\left\{Z_{n}\right\}_{n \geq 1}$ is uniformly integrable.

The next theorem displays the fact that if a process satisfies the hypothesis of Theorem 2.1 and is $\mathrm{MIL}$, then some additional properties of the decomposition are obtained just because the process is MIL.

THEOREM 2.2. Let $\left\{X_{n}\right\}_{n \geq 1}$ be MIL and satisfy the hypothesis of Theorem 2.1. Then $\left\{Z_{n}\right\}_{n \geq 1}$ is MIL and $Z_{n} \rightarrow 0$ a.e.

Proof. That $\left\{Z_{n}\right\}_{n \geq 1}$ is MIL follows immediately from the equality

$$
E\left(X_{n} \mid \mathfrak{U}_{m}\right)-X_{m}=E\left(Z_{n} \mid \mathfrak{A}_{m}\right)-Z_{m}
$$

That $Z_{n} \rightarrow 0$ a.e. follows from the fact that for each $m$,

$$
\sup _{n \geq m}\left|E\left(Z_{n} \mid \mathfrak{A}_{m}\right)-Z_{m}\right| \geq \lim _{\substack{n \rightarrow \infty \\ n \geq m}}\left|E\left(Z_{n} \mid \mathfrak{A}_{m}\right)-Z_{m}\right|=\left|Z_{m}\right|
$$

Merely let $m \rightarrow \infty$ in the previous inequality and the result follows.

It should be observed that Example 2.2 shows that there are processes for which the hypothesis of Theorem 2.1 are satisfied and $\left\{Z_{n}\right\}_{n \geq 1}$ does not approach zero a.e.

This suggests the following definition. 
DEFINITION 2.1. An MIL process $\left\{X_{n}\right\}_{n \geq 1}$ will have a complete Riesz decomposition if it not only has a weak Riesz decomposition but moreover it satisfies:

(i) $\left\{Z_{n}\right\}_{n \geq 1}$ is MIL,

(ii) $Z_{n} \rightarrow 0$ a.e.,

(iii) $\left\{Z_{n}\right\}_{n \geq 1}$ is uniformly integrable and

(iv) $\lim _{n \rightarrow \infty} E\left(Z_{n} \mid \mathfrak{A}_{m}\right)=0$ a.e. for each $m$.

The next few theorems will establish a class of MIL which possesses a complete Riesz decomposition and contains all amarts.

DeFINITION 2.2. Let $\left\{X_{n}\right\}_{n \geq 1}$ be an MIL process with

(i) $\sup _{n \geq m}\left|E\left(X_{n} \mid \mathfrak{A}_{m}\right)\right| \in L_{1}$ for each $m$ and

(ii) for any $\varepsilon>0$ there exist an increasing sequence $\left\{N_{m, \varepsilon}\right\}_{m=1}$ with $N_{m, \varepsilon} \geq m$ for each $m$ and

$$
\varlimsup_{m \rightarrow \infty} \int_{n=N_{m, e}} \sup _{m}\left|E\left(X_{n} \mid \mathfrak{A}_{m}\right)-X_{m}\right|<\varepsilon .
$$

Then, call $\left\{X_{n}\right\}_{n \geq 1}$ an MIL process of class $B$.

Remark. Even though by definition of an MIL it cannot be said that $\lim \int \sup \left|E\left(X_{n} \mid \mathfrak{X}_{m}\right)-X_{m}\right|=0$, in as much as interchange of limit and integral is not always possible, the purpose of Definition 2.2 is to consider MIL's where a (weak) form of the "interchange" is possible. If the process $\left\{X_{n}\right\}_{n \geq 1}$ is martingale to begin with, parts (i) and (ii) of Definition 2.2 follow immediately.

TheORem 2.3. Every MIL of class B has a complete Riesz decomposition.

Proof. The major effort in proving this theorem is to show that $\left\{X_{n}\right\}_{n \geq 1}$ (the MIL process of class $B$ ) is temperable. The other parts will follow from this and also Theorem 2.1 and Theorem 2.2. In addition, it must be proved that $\left\{Z_{n}\right\}_{n \geq 1}$ is uniformly integrable.

To show $\left\{X_{n}\right\}_{n \geq 1}$ is temperable, consider an arbitrary but fixed integer $i$. Let $n>\bar{n} \geq i$ and write

$$
\left|E\left(X_{n} \mid \mathfrak{U}_{i}\right)-E\left(X_{\bar{n}} \mid \mathfrak{A}_{i}\right)\right| \leq E\left[\left(\left|E\left(X_{n} \mid \mathfrak{A}_{m}\right)-X_{m}\right|+\left|E\left(X_{\bar{n}} \mid \mathfrak{X}_{m}\right)-X_{m}\right|\right) / \mathfrak{A}_{i}\right]
$$

for any $m \geq i$ and $\bar{n} \geq m$.

Define

$$
f_{m} \equiv \sup _{n, \bar{n}>m}\left|E\left(X_{n} \mid \mathfrak{A}_{i}\right)-E\left(X_{\bar{n}} \mid \mathfrak{A}_{i}\right)\right|
$$

Then,

$f_{m} \geq 0$ each $m$,

$f_{m} \in L_{1}$ each $m$,

$f_{m}$ is $\mathfrak{A}_{i}$ measurable for each $m$, and

$f_{m}$ is decreasing in m.a.e. 
To show $f_{m} \rightarrow 0$ a.e. it will be sufficient to show that a subsequence goes to zero a.e. From the first inequality of this proof it follows that

Thus

$$
f_{N_{m, e}} \leq E\left[\sup _{n, \bar{n}>N_{m, e}}\left(\left|E\left(X_{n} \mid \mathfrak{A}_{m}\right)-X_{m}\right|+\left|E\left(X_{\bar{n}} \mid \mathfrak{A}_{m}\right)-X_{m}\right|\right) / \mathscr{X}_{i}\right]
$$

$$
\varlimsup_{m \rightarrow \infty} \int f_{N_{m, a}} \leq 2 \varlimsup_{m \rightarrow \infty} \int \sup _{n>N_{m, s}}\left|E\left(X_{n} \mid \mathfrak{A}_{m}\right)-X_{m}\right| \leq 2 \varepsilon .
$$

Since $\int f_{m}$ is a decreasing sequence, the previous inequality gives that for $\varepsilon>0$ there exists a sufficiently large $M(\varepsilon)$ such that for $m>M(\varepsilon), \int f_{m}<\varepsilon$. Thus $\int f_{m} \rightarrow 0$ as $m \rightarrow \infty$ and there exists a subsequence converging to zero a.e. and so $\left\{X_{n}\right\}_{n \geq 1}$ is temperable.

Next it will be proved that $\left\{Z_{n}\right\}_{n \geq 1}$ is uniformly integrable. Choose $\varepsilon>0$; then

$$
\varlimsup_{m \rightarrow \infty} \int \sup _{n>N_{m, e}}\left|E\left(Z_{n} \mid \mathfrak{A}_{m}\right)-Z_{m}\right|<\varepsilon ;
$$

that is, for $m \geq M(\varepsilon), \int \sup _{n \geq N_{\text {m. }}}\left|E\left(Z_{n} \mid \mathfrak{A}_{m}\right)-Z_{m}\right|<\varepsilon$. For $m<M(\varepsilon)$, there exists a $\delta>0$ so that for any set with $P(A)<\delta, \int_{A}\left|Z_{m}\right|<\varepsilon$. For $m \geq M(\varepsilon)$, the same $\delta$ will suffice in as much as for $n \geq N_{m, \varepsilon}$

$$
\int_{\mathrm{A}}\left|E\left(Z_{n} \mid \mathfrak{A}_{m}\right)-Z_{m}\right| \leq \int_{n \geq N_{m, \varepsilon}}\left|E\left(Z_{n} \mid \mathfrak{A}_{m}\right)-Z_{m}\right|<\varepsilon .
$$

Merely let $n \rightarrow \infty$ in the previous inequality and get $\int_{A}\left|Z_{m}\right|<\varepsilon$ for $m \geq M(\varepsilon)$. It is also clear that $\sup \int\left|Z_{m}\right|<\infty$ and so $\left\{Z_{n}\right\}_{n \geq 1}$ is uniformly integrable.

The next theorem presents a converse to Theorem 2.3 .

THEOREM 2.4. Let $\left\{X_{n}\right\}_{n \geq 1}$ be an $M I L$ with $\sup _{n \geq m}\left|E\left(X_{n} \mid \mathfrak{Q}_{m}\right)\right| \in L_{1}$ for each $m$ and with a complete Riesz decomposition. Then $\left\{X_{n}\right\}_{n \geq 1}$ is an MIL of class $B$.

Proof. Since $\left\{X_{n}\right\}_{n \geq 1}$ has a complete Riesz decomposition, it is immediate that $\sup _{n \geq m}\left|E\left(Z_{n} \mid \mathfrak{A}_{m}\right)\right| \in L_{1}$ for each $m, \lim _{n \rightarrow \infty} E\left(Z_{n} \mid \mathfrak{A}_{m}\right)=0$ a.e. for each $m$ and $Z_{n} \rightarrow 0$ in $L_{1}$. Choose $\varepsilon>0$. Then there exists a sufficiently large $M$ so that for $m \geq M, \int\left|Z_{m}\right|<\varepsilon$. For each $m$, select $N_{m, \varepsilon}$ so that for all $n \geq N_{m, \varepsilon}$ we have $\left|E\left(Z_{n} \mid \mathfrak{A}_{m}\right)\right|<\varepsilon$ on a set $A_{m}$ with $P\left(A_{m}^{C}\right)$ so small that $\int_{A_{m}^{c}}^{c} \sup _{n \geq m}\left|E\left(Z_{n} \mid \mathfrak{A}_{m}\right)\right|<\varepsilon$. Thus, for $m \geq M$,

$$
\int \sup _{n \geq N_{m, s}}\left|E\left(Z_{n} \mid \mathscr{U}_{m}\right)-Z_{m}\right| \leq \int_{\mathcal{A}_{m}} \sup _{n \geq N_{m, e}}\left|E\left(Z_{n} \mid \mathfrak{U}_{m}\right)+\int_{\mathbb{A}_{m}^{c}} \sup _{n \geq m}\right| E\left(Z_{n} \mid \mathfrak{A}_{m}\right)\left|+\int\right| Z_{m} \mid \leq 3 \varepsilon .
$$

Thus,

$$
\varlimsup_{m \rightarrow \infty} \int \sup _{n \geq N_{m, e}}\left|E\left(Z_{n} \mid \mathfrak{U}_{m}\right)-Z_{m}\right|<3 \varepsilon
$$

where $\varepsilon$ was arbitrary, the proof is complete. 
THEOREM 2.5. Every amart is MIL of class B.

Proof. Every amart is MIL; see [3] and [6]. Every amart satisfies the hypothesis of Theorem 2.4; see [5], p. 209].

Remark 2.1. The family of MIL of class $B$ strictly contains amarts. See Example 2.1. For some particulars, see [6, p. 318].

REMARK 2.2. In [10] it is proved that $\left\{X_{n}\right\}_{n \geq 1}$ is amart if and only if $X_{n}=Y_{n}+Z_{n}$, where $\left\{Y_{n}\right\}_{n \geq 1}$ is a martingale and $\left|Z_{n}\right| \leq S_{n}$ a.e., where $\left\{S_{n}\right\}_{n \geq 1}$ is a positive supermartingale which converges to zero in $L_{1}$. This result shows that

$$
\lim _{m \rightarrow \infty} \int_{n \geq m} \sup _{n \geq m}\left|E\left(X_{n} \mid \mathfrak{X}_{m}\right)-X_{m}\right|=0
$$

and so condition (ii) of Definition 2.2 is easily satisfied. This, in a qualitative sense suggests how much larger MIL's of class $B$ are than the class of amarts.

REMARK 2.3. Recall that a process $\left\{X_{n}\right\}_{n \geq 1}$ is semi amart if and only if $\sup _{\tau \in T}\left|E\left(X_{\tau}\right)\right|<$ $\infty$ where $T$ is the set of all bounded stopping times. Example 2.1 displays a process which is not semi amart but is MIL of class $B$; see [6, p. 318] for details. Hence, MLL's of class $B$ contain processes which are not semi amart. It is obvious from [10], Theorem 1 that there are semi amarts which are not MIL of class $B$.

This section will conclude with a lemma which will give direct access to the fact that every quasi martingale is a martingale in the limit of class $B$.

PRoposition 2.6. Every positive supermartingale is a martingale in the limit of class $B$.

Proof. Let $\left\{X_{n}\right\}_{n \geq 1}$ be a positive super martingale with $X$ as its a.e. limit. Observe that for any $m,\left\{E\left(X_{n} \mid \mathfrak{U}_{m}\right)\right\}_{n \geq m}$ has $E\left(X \mid \mathfrak{U}_{m}\right)$ as an a.e. limit and for each $m$ is an a.e. decreasing sequence in $n$. Hence,

$$
\sup _{n \geq m}\left|E\left(X_{n} \mid \mathfrak{A}_{m}\right)-X_{m}\right| \leq X_{m}-E\left(X \mid \mathfrak{A}_{m}\right)
$$

for each $m$. With this inequality at hand it is at once clear that the process is martingale in the limit and moreover that it satisfies the conditions of Definition 2.2; in particular property (ii) of Definition 2.2 is satisfied with $N_{m, e}=m$ for each $m$ and $\varepsilon$.

THEOREM 2.7. Every quasi martingale is a martingale in the limit of class $B$.

Proof. Every quasi martingale can be expressed as the difference of two positive supermartingales. This in conjuction with Proposition 2.6 proves the result.

Acknowledgement. The author would like to thank Professor R. Syski of the University of Maryland for pointing out Proposition 2.6. 


\section{REFERENCES}

1. L. Baez-Duarte, An a.e. divergent martingale that converges in probability, J. Math. Anal. Appl. 36 (1971), 149-150.

2. L. H. Blake, A note concerning the $L_{1}$ convergence of a class of games which become fairer with time, Glasgow Math. J. 13 (1972), 39-41. $381-384$

3. L. H. Blake, Every amart is a martingale in the limit, J. London Math. Soc. 18 (1978),

4. J. L. Doob, Stochastic processes (Wiley, 1953).

5. G. A. Edgar, and L. Sucheston, Amarts: a class of asymptotic martingales, J. Multivariate Anal. 6 (1976), 193-221.

6. G. A. Edgar, and L. Sucheston, Martingales in the limit and amarts. Proc. Amer. Math. Soc. 67 (1977), 315-319.

7. P. A. Meyer, Probability and potentials (Waltham, Mass., 1966).

8. A. G. Mucci, Limits for martingale-like sequences, Pacific J. Math. 48 (1973), 197-302 $539-541$.

9. A. G. Mucci, Another martingale convergence theorem, Pacific J. Math. 64 (1976),

10. L. Sucheston and N. Ghoussoub, A refinement of the Riesz decomposition for amarts and semiamarts, J. Multivariate Anal. 8 (1978), 146-150.

College of Staten Island

CUNY

NEW YORK 10301 